\title{
Cyclic thrombocytopenia in a patient with polycythemia vera: a case report
}

\author{
Ilja Depoortere ${ }^{1}$, Vincent Maertens ${ }^{2}$, Marieke Criel ${ }^{1}$, Marleen Vanden Driessche ${ }^{3}$, Inge Geerts ${ }^{1}$ \\ ${ }^{1}$ Laboratory Medicine, Imelda Hospital, Bonheiden, Belgium \\ ${ }^{2}$ Clinical Hematology, Imelda Hospital, Bonheiden, Belgium \\ ${ }^{3}$ Laboratory Medicine, Rode Kruis-Vlaanderen (HILA), Mechelen, Belgium

\section{OBJECTIVES}

We present a case of a 73-year-old patient with polycythemia vera $(P V)$, in whom strong fluctuations of platelet counts (ranging from 31 to $1334 \times 10^{3} / \mu \mathrm{L}$ ) were noticed after onset of hydroxyurea $(\mathrm{HU})$ therapy. This cyclic oscillation in platelet counts, has been sporadically described in myeloproliferative neoplasms (MPN), especially in PV.

\section{METHODS}

Platelet measurements were performed by impedance, fluorescence and optic methods on EDTA and on citrated whole blood (XN-3000®), Sysmex) and confirmed with manual microscopic count (number of platelets/ 1000 red blood cells) on a few samples. Some measurements were checked on another analyzer (DxH®, Beckman Coulter) with concordant results.

During follow-up three bone marrow biopsies were examined independently of the platelet count. All biopsies showed a hypercellular bone marrow with normal to strong representation of the megakaryocytic lineage.

\section{RESULTS}

Fluctuations in platelet count in our patient started immediately after onset of $\mathrm{HU}$ therapy, and were possibly enforced by multiple dose adjustments. Maintaining constant dose resulted in a more narrow amplitude of the oscillations. (figure 1) Because of evolution to myelofibrosis (enlarged spleen, increase in fibrosis and blastosis in bone marrow) the decision was made to switch therapy to ruxolitinib. Platelet count remained stable hereafter.

In literature provoked and unprovoked oscillations in platelet numbers have been described. Unprovoked oscillations are probably attributed to an unstable hematopoietic stem cell pool. Transient $\mathrm{HU}$ induced depletion of megakaryocytes could provoke fluctuations followed by a compensatory thrombopoietin (TPO)-induced stimulation of megakaryocytes and precursors. ${ }^{[1,2]}$

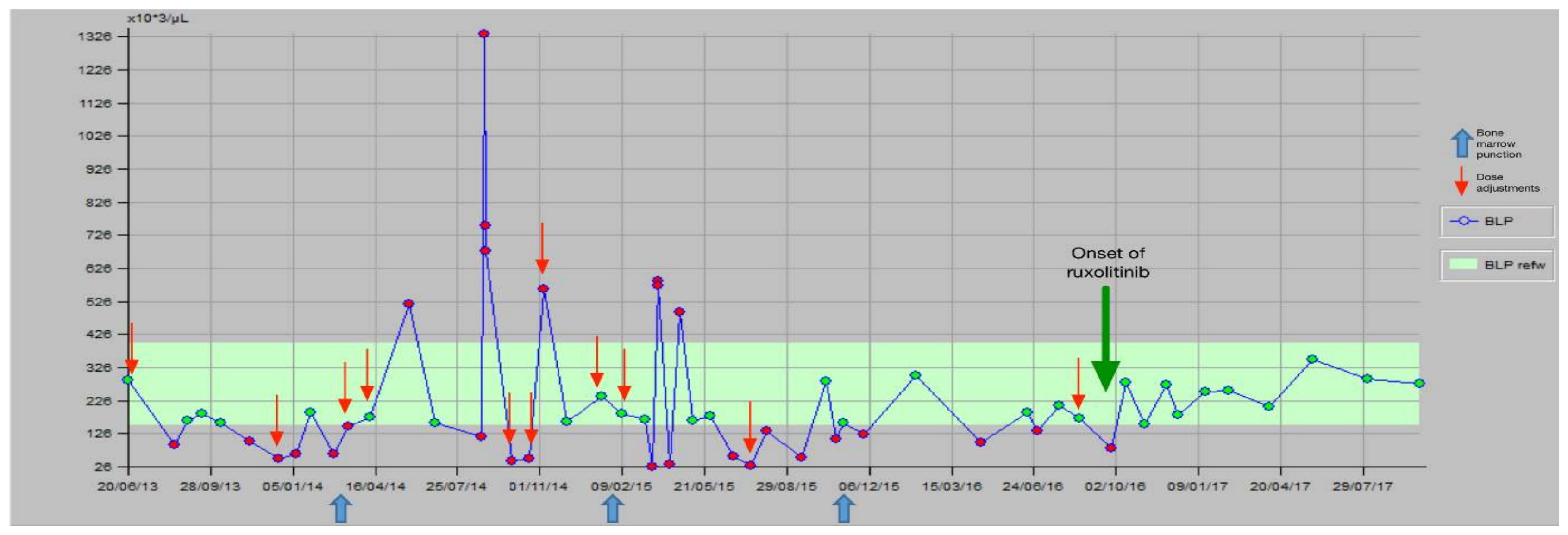

Figure 1: History of platelet count. Dose adjustments of hydroxyurea, bone marrow biopsies and onset of ruxolitinib were marked.

\section{CONCLUSION}

Cyclic thrombocytopenia is a rare finding and should be considered in HU-treated MPN patients. We share the experience that frequent dose adjustments may provoke a bouncing ball effect on platelet count. ${ }^{[1]}$ Some patients benefit from maintaining the therapy at a constant dose, others require withholding or switching therapy. ${ }^{[1,3]}$ Thus, intensive follow-up and personalized therapy management is mandatory.

${ }^{3}$ Burthem J, Chaudhry MS. Hydroxycarbamide associated platelet count oscillations in a patient with polycythaemia vera. A case report and review of the literature. Platelets 2008 ; 